\title{
Structure equation as novel strategy for optimum reflector design
}

\author{
Chung-Jen Ou
}

Hsiuping Solar Observatory and Photo-Chemical Lab., Department of Energy and Materials Technology, Hsiuping University of Science and Technology, No. 11, Gongye Rd., 40128 Dali, Taichung, Taiwan ROC; e-mail: crou@hust.edu.tw

\begin{abstract}
The optimal use of energy resources is the central dogma for green technology and bio-optics. Reflectors provide a simple and fundamental structure for energy transport from a light emitting diode (LED) chip, and an appropriately designed reflector can reduce the fabrication cost of secondary optics for the LED. This paper demonstrates the role of three proposed reflector geometric factors in the two performance metrics - uniformity and collected energy (power), for designing LED reflectors. Through canonical factor analysis, a linear structure equation for LED reflector is suggested, and the methodology for designing the optimal shape is discussed. In addition, a generalized factor and a synthesis response are proposed for a more comprehensive investigation of optical performance. Results indicate a key parameter for balancing the optical performance, and the effects of various parameters and the trade-offs are revealed.
\end{abstract}

Keywords: illumination design, light-emitting diodes, structure equation.

\section{Introduction}

For the designing of the LED secondary lens, the effects of the reflectors should be known to the optical designers, such that the appropriate lens design can be integrated with the reflectors. However, the characteristics and the optical behaviors of the reflector for fabrication are not perfectly understood. In most cases, although the energy emitted from the side surface of an LED die comprises part of the total energy, it can still change the spatial and the angular distribution of the emitted light, leading to a change in the large-scale uniformity of the system. For this reason, one should focus on establishing a procedure to explore the stand-alone effects of LED reflectors for energy distribution on targeting surfaces. The comparison between the secondary optics and the reflectors is exactly analogous to refractive and reflective optics, and the advantage of using the appropriate reflector is that it is free from wavelength dispersion. The reflector can modify the original intensity pattern of the LED die more easily in the first stage than the techniques of secondary optical lens [1], therefore the reflector plays an important role in energy distribution [2-4]. However, few studies have examined the design cri- 
teria and limitations of the LED reflector, and how various geometric factors affect their behaviors [ $\underline{5}-\underline{8}]$. One can see that a reflector can bend the side-emitted rays, resulting in two groups of distributions in the far-field patterns [9], and the distance between these two distributed energy regions will vary with the parameters of the reflectors. Another fact revealed in the literature is that the reflector acts like a focusing mirror with a very short effective focal length, leading to a dramatic change in the energy spreading patterns after a projection distance $[\underline{7}, \underline{9}]$. The capabilities of bending, focusing, and diverting the rays clearly depends on the geometric factors of the reflector. On the other hand, the additional optical encapsulant and coverage material will influence the performance of LED module [10, 11], hence it is of importance to draw a so-called "baseline" for the solely combination between the LED die and the reflector. As a summary, since the understanding of the baseline of the LED module (LED die with a reflector) is important in every way, the purpose of this research is to propose the appropriate shape factors and the phenomenological structures of LED reflectors to study the performance (e.g., uniformity and energy distribution) by canonical factor analysis [12-15]. Although methods such as analysis of variance (ANOVA) work with Taguchi method [12] can be helpful in identifying the principal variables among these geometric factors, however, the present study is to reveal the structure equation, rather than only to analyze factors for quality control.

\section{Theory and methods}

\subsection{System configurations}

Figure 1 is the LED structure with a typical reflector. Parameters of the LED structure include the radius of the reflector $R$, the inner radius $r$, the length of the rim surface $L$, the height of the reflector base $h$, height of the total reflector $H$, the height of the LED die $h_{\mathrm{LED}}$, and the angle of the reflector $\theta$. Radius $r$, height $H$, and the angle $\theta$ can affect the distribution of the LED apodization, and this reflector is meant to be optimized for geometric parameters that produce the best performance. To explore these properties,
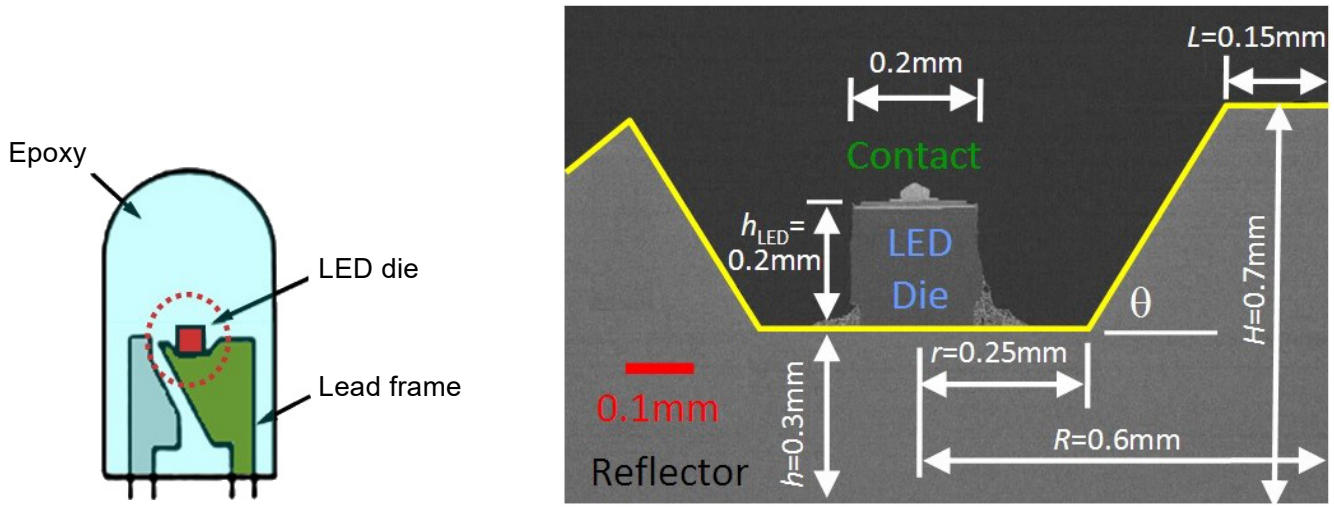

Fig. 1. Typical LED structure with reflector profile. 


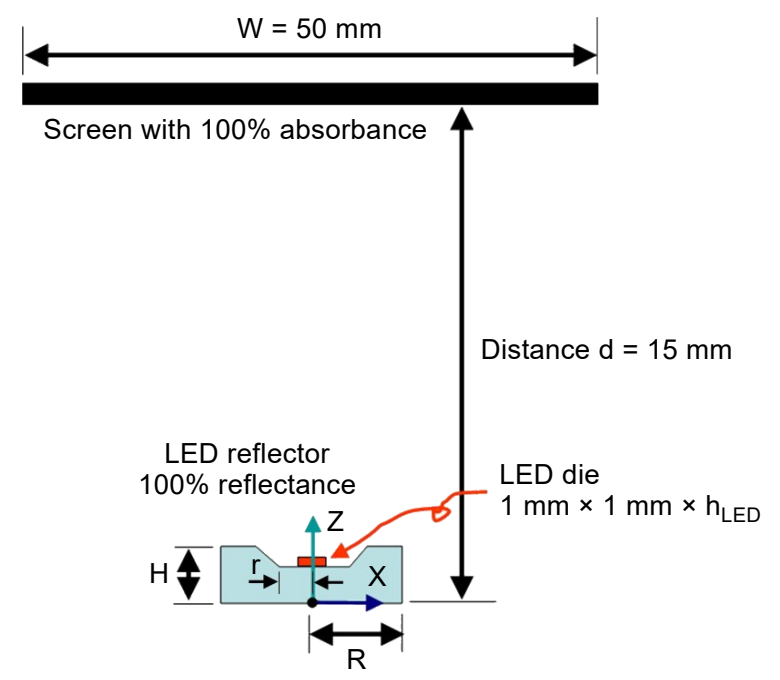

Fig. 2. Illuminating system configuration for the present study.

appropriate modelling is required [16]. Here the optical software Light-Tool [17] is adopted for establishing the optical model, with the solid based entities (such as the SAT format of the 3D ACIS Modeler) and it can be transferred easily into a finite element analysis program for thermal-structural investigation $[\underline{9}, \underline{18}]$. Figure 2 shows the illuminating configuration of the present experiment, and the dimension of LED die is predetermined. Note that the encapsulant is being removed to investigate the effects of the reflector only. The present model can then determine the collection efficiency for various reflector shapes. A surface-emitting model with five emitting surfaces simulates the emission of the LED die, where it is considered that the entire surfaces are defect free. Meanwhile, the screen surface property is $100 \%$ absorbance, and the reflectance of the reflector surface is $100 \%$. The angular apodization of the LED die is presumed to be Lambertian, and the spatial apodization of the LED surface is assumed uniform. These assumptions are not perfectly real, but constitute a very practical first -order approach. The advantage of these assumptions is that they enable the fabricators to evaluate the baseline of the LED system performance, since the purpose of the present study is to identify the optimum criteria for different geometric configurations.

\subsection{Reflector parameters}

To evaluate the optical performance of the reflector, Fig. 3a describes the geometry of the reflector with six initial parameters: $r, R, H, \theta, h$ and $L$. Note that the dimension of the LED die is $1 \mathrm{~mm} \times 1 \mathrm{~mm} \times h_{\mathrm{LED}}$, where the height of the LED die $h_{\mathrm{LED}}$ is assigned to $0.1 \mathrm{~mm}$. The angle $\theta$ can be obtained by the following equation:

$$
\theta=\tan ^{-1}\left(\frac{H-h}{R-r-L}\right)
$$




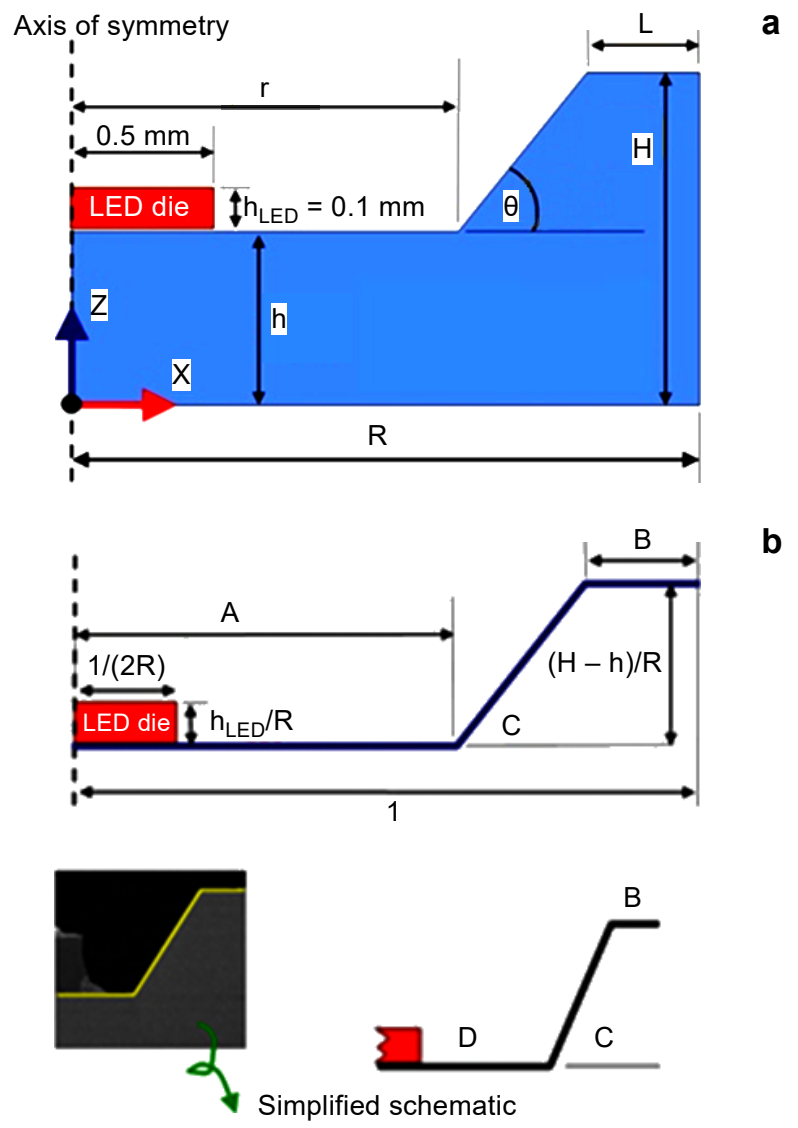

Fig. 3. Initial geometric parameters of a reflector profile (a). Reduced geometric parameter set $(B, C, D)$ for describing the reflector $(\mathbf{b})$.

However, it is important to see if there is any possibility to reduce the number of these initial geometric parameters. The first step is to consider $R$ as the reference length, and the second step is to realize that from the optical point of view, the thickness $h$ is not directly relevant to optical performance. Therefore, Fig. $3 \mathbf{b}$ is the reduced system with three defined factors.

Note that after taking the radius $R$ as the reference, then the dimensionless parameters $A, B$, and $C$ are defined as:

$$
\begin{aligned}
& A \equiv r / R \\
& B \equiv L / R \\
& C \equiv \tan \theta
\end{aligned}
$$

Assignments for $A, B$, and $C$ can complete a set of definitions for a unique reflector design. In a previous report [9], $(R-r)$ is introduced as the dimensionless references. 
However, such treatment leads to inconveniences due to the lack of appropriate physical interpretations. Therefore, a new definition would be much better to describe the geometric features of the reflector:

$$
\begin{aligned}
& C \equiv \tan \theta=\frac{(H-h) / R}{1-r / R-L / R}=\frac{(H-h) / R}{1-A-B} \\
& D \equiv \frac{1}{1-A}
\end{aligned}
$$

Now one can denote the three reduced geometric factors as $B, C$, and $D$, where the physical meaning of the factor $D$ is the ratio between the outer radius $R$ and the difference between the two radii $(R-r)$. Since $0 \leq B \leq(1-A)$ and $H>h$, the following factor space stands:

$$
\begin{aligned}
& 0 \leq B \leq 1 \\
& 0 \leq C \leq \infty \\
& 1 \leq D \leq \infty
\end{aligned}
$$

Two more things need to be mentioned for these factors. The first thing is that it was expected that the effects of $B$ are less sensitive to the results which are due to the fact that $B$ represents the edge surface of the reflector. In fact, $B$ is a parameter affecting the dominant factor $C$, such that it contributes to the variations of the optical metrics. The second thing is that there are two extreme examples for the angle factor $C$-vertical reflector wall condition $\left(\theta=90^{\circ} \Rightarrow C \rightarrow \infty\right)$ and the horizontal reflector wall condition $\left(C=0 \Rightarrow \theta=0^{\circ}\right)$, both indicate that there is no any side-emitting rays which can target to the detecting surface.

\subsection{Performance metrics}

The uniformity $U$ and the total power $P$ are the two most frequently considered metrics for non-imaging optical system. However, it is acknowledged that usually these metrics conflict with each other, due to limitations from both the physical principles and engineering restrictions. Hence, to take the best advantages of the defining geometric factors, the construction of the performance metrics for the merit function becomes critical. The configuration on the detecting screen and a typical illuminating pattern for the analysis are shown in Fig. 4. In most situations, the diameter of the circular illuminating region $2 R_{\mathrm{S}}$ will not be exactly the same as the length of the screen $W$. However, for the designing of the non-imaging simulation merit function, the performance of an ideal solution can be measured by the energy distribution on a rectangle (square area in this report) plane with the illuminating patterns within. The best circumstance is that the illuminating circular region (with a defined fall off edge) is exactly happening to be in the target area $\left(R_{\mathrm{S}}=W / 2\right)$. This is the largest illuminating area 


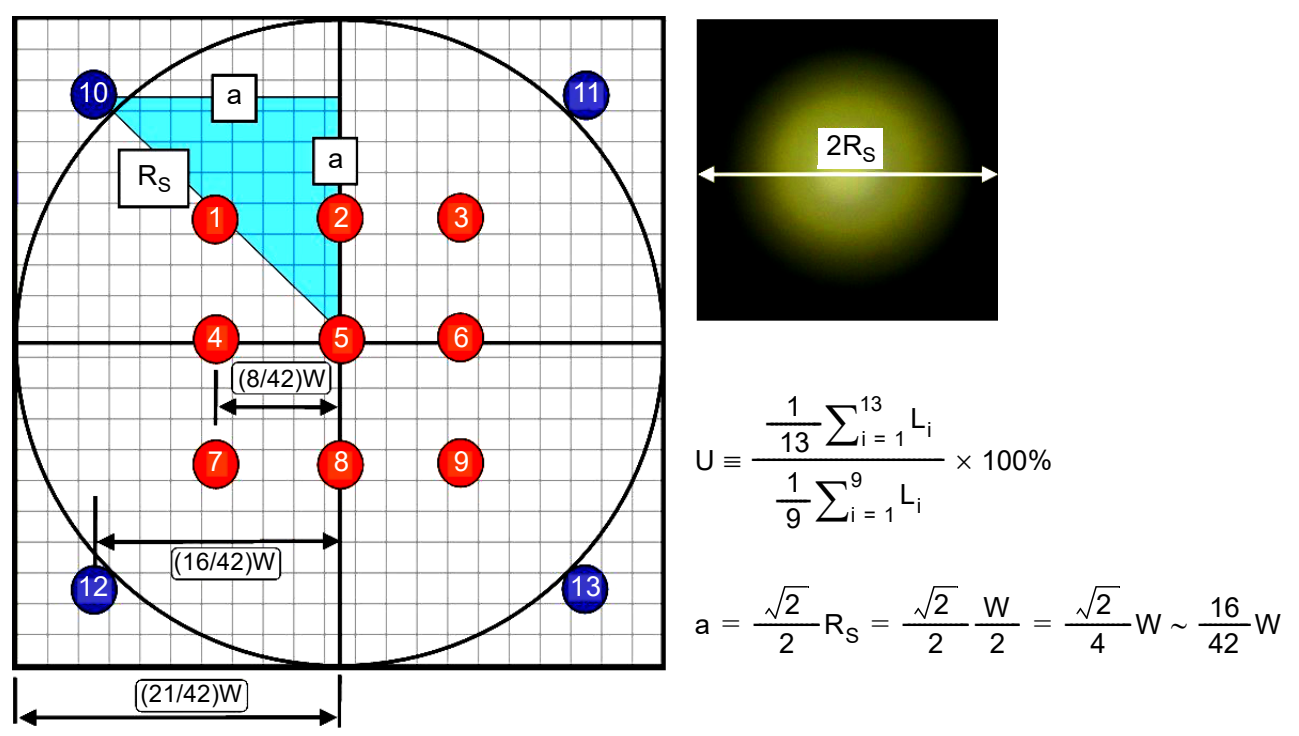

Fig. 4. Detecting screen and the definition of the positions for 13 sensing points.

under tolerable energy loss. Hence, the detecting point can be arranged as shown in the left part of Fig. 4.

The uniformity $U$ for the present study can be defined by taking the 13 measurement points on the detecting screen,

$$
U \equiv \frac{\frac{1}{13} \sum_{i=1}^{13} L_{i}}{\frac{1}{9} \sum_{i=1}^{9} L_{i}} \times 100 \%
$$

where $L_{i}$ is the illuminance on the $i$-th measurement points. The advantage of such definitions is that it can evaluate the two dimensional energy distributions directly. Other types of uniformity definitions might work as well, since there are no limitations of the user defined performance metrics for the demonstration of the present structure equation method.

In Fig. 4 the detecting screen is divided into $21 \times 21$ grids, and the characteristic lengths for these detecting points are $(8 / 42) W$ and $(16 / 42) W$. Note that $a=\sqrt{2} W / 4$, and the selection of the approximation for $a \approx(16 / 42) W \approx 0.38095 W$. Although $14 / 42 \approx 0.33333$ is much closer to the $\sqrt{2} / 4 \approx 0.35355$, however, since the energy will dramatically change outside the edge of the illuminating region, this report recommends to put the four detecting points outside the circles for capturing the most sensitive part of the largest illuminating conditions. Due to the nature of the cosine fourth law, it is expected that the average for the illuminance of all 13 points is less than the 
average of the 9 points (this assures that $U<1$ ). As for another performance metric $P$, the calculation of the total power $P$ illuminating on the screen is quite straightforward. The total power $P$ on the target plane is directly obtained from the ray tracing report, which is independent of the corresponding bin number (pixel size on the receivers). To ensure the effectiveness of the simulation results, the error estimate at peak (EEP) [17] that is smaller than $5 \%$ for each simulation is being verified.

\subsection{Analysis procedures}

To complete the factor analysis, the three factors $B, C$, and $D$ are arranged into four levels, such that a total of $n=4^{3}=64$ experiments are required. In fact, the number of experiments can be reduced by $L_{n}\left(4^{3}\right)$ orthogonal Latin squares to $n=32$ or $n=16$ $[\underline{13}-15,19]$. However, such analysis procedures are not the primary issues of the present report. This report proceeds with the complete set of 64 experiments since the cost for generating this information is affordable by the AMD Athlon X2 processor. The four levels of the factor $B$ are $0.05,0.1,0.15$, and 0.2 ; the four levels of the $C$ factor are $0.8391,1,1.1918$, and 1.4281; and the four levels of the factor $D$ are 2.5, 2.8571, 3.3333 , and 4 . These values are derived from the variations of reflector geometric parameters $A=0.6,0.65,0.7,0.75$ and $B=0.05,0.1,0.15,0.2$. In addition, the $C$ values are calculated from $\theta=40^{\circ}, 45^{\circ}, 50^{\circ}$, and $55^{\circ}$. The total number of rays is 1000000 , the unit of the power on the screen is the watt (W), and the total power of the LED is taken as $1 \mathrm{~W}$. For steady state emitting, the equivalent between the energy and the power required only a time factor. With these values, the simulation results are automatically presented as ratios expressed in percentages. As mentioned before, the entire EEP during ray tracing simulation is less than $5 \%$. However, variations between the results still required statistical analysis.

\section{Results and discussions}

A series of numerical values were derived according to previous equations, staring with demonstrated LED design $R=1 \mathrm{~mm}$, height of the LED die $h_{\mathrm{LED}}=0.1 \mathrm{~mm}$ with the foundation of the LED die as $1 \mathrm{~mm}$ square. This contribution to the energy given off from each side was $7.14 \%$ of the total energy. The energy from the side-emitting surface comprises around $28.6 \%$ of the total energy, and the top surface emitted $71.4 \%$ of the total energy. During the experiments, given a set of design parameters $(B, C, D)$, the coordinates of the reflector can be constructed. As mentioned before, the reflector is $100 \%$ reflective without any scattering mechanisms in this model, such that the upper limits of the reflector power $P$ performance can be expected (not necessary the uniformity $U$ ). As a preliminary check, energy balancing was confirmed by calculating the energy from each emitting surface without a reflector. The square plane detector, as shown in Fig. 2 for receiving the emitting energy, is located $d=15 \mathrm{~mm}$ away from the LED source, and the size of the detecting screen is $W=50 \mathrm{~mm}$ square. Tables $1-4$ 
T a b l e 1. Experimental results for $B=0.05$, and $\theta=40^{\circ}(C=0.8391), \theta=45^{\circ}(C=1), \theta=50^{\circ}$ $(C=1.1918)$, and $\theta=55^{\circ}(C=1.4281)$.

\begin{tabular}{lllll}
\hline$B$ & $C=\tan \theta$ & $D$ & $P[\mathrm{~W}]$ & $U[\%]$ \\
\hline 0.05 & 0.8391 & 2.5000 & 0.82482 & 72.01 \\
0.05 & 0.8391 & 2.8571 & 0.83195 & 72.31 \\
0.05 & 0.8391 & 3.3333 & 0.82177 & 72.40 \\
0.05 & 0.8391 & 4.0000 & 0.79852 & 72.61 \\
0.05 & 1.0000 & 2.5000 & 0.84639 & 71.51 \\
0.05 & 1.0000 & 2.8571 & 0.85729 & 71.91 \\
0.05 & 1.0000 & 3.3333 & 0.84578 & 72.23 \\
0.05 & 1.0000 & 4.0000 & 0.81787 & 72.42 \\
0.05 & 1.1918 & 2.5000 & 0.86627 & 71.10 \\
0.05 & 1.1918 & 2.8571 & 0.88316 & 71.24 \\
0.05 & 1.1918 & 3.3333 & 0.87284 & 71.75 \\
0.05 & 1.1918 & 4.0000 & 0.84159 & 72.04 \\
$\mathbf{0 . 0 5}$ & $\mathbf{1 . 4 2 8 1}$ & $\mathbf{2 . 5 0 0 0}$ & $\mathbf{0 . 8 8 3 2 0}$ & $\mathbf{7 0 . 7 4}$ \\
$\mathbf{0 . 0 5}$ & $\mathbf{1 . 4 2 8 1}$ & $\mathbf{2 . 8 5 7 1}$ & $\mathbf{0 . 9 0 7 6 2}$ & $\mathbf{7 1 . 0 3}$ \\
0.05 & 1.4281 & 3.3333 & 0.90019 & 71.25 \\
0.05 & 1.4281 & 4.0000 & 0.82168 & 72.40 \\
\hline
\end{tabular}

$\mathrm{T}$ a b 1 e 2. Experimental results for $B=0.10$, and $\theta=40^{\circ}(C=0.8391), \theta=45^{\circ}(C=1), \theta=50^{\circ}$ $(C=1.1918)$, and $\theta=55^{\circ}(C=1.4281)$.

\begin{tabular}{lllll}
\hline$B$ & $C=\tan \theta$ & $D$ & $P[\mathrm{~W}]$ & $U[\%]$ \\
\hline 0.10 & 0.8391 & 2.5000 & 0.81185 & 72.10 \\
$\mathbf{0 . 1 0}$ & $\mathbf{0 . 8 3 9 1}$ & $\mathbf{2 . 8 5 7 1}$ & $\mathbf{0 . 8 1 7 3 0}$ & $\mathbf{7 2 . 4 0}$ \\
0.10 & 0.8391 & 3.3333 & 0.80546 & 72.61 \\
0.10 & 0.8391 & 4.0000 & 0.78014 & 72.73 \\
0.10 & 1.0000 & 2.5000 & 0.83033 & 72.00 \\
0.10 & 1.0000 & 2.8571 & 0.83888 & 72.00 \\
0.10 & 1.0000 & 3.3333 & 0.82495 & 72.34 \\
0.10 & 1.0000 & 4.0000 & 0.79459 & 72.59 \\
0.10 & 1.1918 & 2.5000 & 0.84922 & 71.80 \\
0.10 & 1.1918 & 2.8571 & 0.86236 & 71.50 \\
0.10 & 1.1918 & 3.3333 & 0.84839 & 71.98 \\
0.10 & 1.1918 & 4.0000 & 0.81414 & 72.34 \\
0.10 & 1.4281 & 2.5000 & 0.86688 & 71.20 \\
0.10 & 1.4281 & 2.8571 & 0.88656 & 71.30 \\
0.10 & 1.4281 & 3.3333 & 0.87442 & 71.67 \\
0.10 & 1.4281 & 4.0000 & 0.83291 & 72.14 \\
\hline
\end{tabular}


T a b 1 e 3. Experimental results for $B=0.15$, and $\theta=40^{\circ}(C=0.8391), \theta=45^{\circ}(C=1), \theta=50^{\circ}$ $(C=1.1918)$, and $\theta=55^{\circ}(C=1.4281)$.

\begin{tabular}{|c|c|c|c|c|}
\hline $\bar{B}$ & $C=\tan \theta$ & $D$ & $P[\mathrm{~W}]$ & $U[\%]$ \\
\hline$\overline{0.15}$ & 0.8391 & 2.5000 & 0.79650 & 72.18 \\
\hline 0.15 & 0.8391 & 2.8571 & 0.80087 & 72.57 \\
\hline 0.15 & 0.8391 & 3.3333 & 0.78772 & 73.00 \\
\hline 0.15 & 0.8391 & 4.0000 & 0.75094 & 71.78 \\
\hline 0.15 & 1.0000 & 2.5000 & 0.81234 & 71.95 \\
\hline 0.15 & 1.0000 & 2.8571 & 0.81735 & 72.80 \\
\hline 0.15 & 1.0000 & 3.3333 & 0.81108 & 73.00 \\
\hline 0.15 & 1.0000 & 4.0000 & 0.76689 & 72.79 \\
\hline 0.15 & 1.1918 & 2.5000 & 0.82933 & 71.35 \\
\hline 0.15 & 1.1918 & 2.8571 & 0.83822 & 71.78 \\
\hline 0.15 & 1.1918 & 3.3333 & 0.82011 & 72.00 \\
\hline 0.15 & 1.1918 & 4.0000 & 0.78214 & 72.69 \\
\hline 0.15 & 1.4281 & 2.5000 & 0.84669 & 71.55 \\
\hline 0.15 & 1.4281 & 2.8571 & 0.86056 & 71.58 \\
\hline 0.15 & 1.4281 & 3.3333 & 0.83759 & 72.00 \\
\hline 0.15 & 1.4281 & 4.0000 & 0.79609 & 72.54 \\
\hline
\end{tabular}

T a b 1 e 4 . Experimental results for $B=0.20$, and $\theta=40^{\circ}(C=0.8391), \theta=45^{\circ}(C=1), \theta=50^{\circ}$ $(C=1.1918)$, and $\theta=55^{\circ}(C=1.4281)$.

\begin{tabular}{|c|c|c|c|c|}
\hline $\bar{B}$ & $C=\tan \theta$ & $D$ & $P[\mathrm{~W}]$ & $U[\%]$ \\
\hline 0.20 & 0.8391 & 2.5000 & 0.78035 & 72.35 \\
\hline 0.20 & 0.8391 & 2.8571 & 0.78301 & 72.90 \\
\hline 0.20 & 0.8391 & 3.3333 & 0.78450 & 73.01 \\
\hline 0.20 & 0.8391 & 4.0000 & 0.71983 & 73.22 \\
\hline 0.20 & 1.0000 & 2.5000 & 0.79202 & 72.07 \\
\hline 0.20 & 1.0000 & 2.8571 & 0.79393 & 73.00 \\
\hline 0.20 & 1.0000 & 3.3333 & 0.77405 & 72.81 \\
\hline 0.20 & 1.0000 & 4.0000 & 0.73041 & 73.10 \\
\hline 0.20 & 1.1918 & 2.5000 & 0.80527 & 71.66 \\
\hline 0.20 & 1.1918 & 2.8571 & 0.81013 & 72.60 \\
\hline 0.20 & 1.1918 & 3.3333 & 0.78750 & 72.61 \\
\hline 0.20 & 1.1918 & 4.0000 & 0.74021 & 73.12 \\
\hline 0.20 & 1.4281 & 2.5000 & 0.82173 & 71.51 \\
\hline 0.20 & 1.4281 & 2.8571 & 0.82497 & 72.50 \\
\hline 0.20 & 1.4281 & 3.3333 & 0.79990 & 72.31 \\
\hline 0.20 & 1.4281 & 4.0000 & 0.74867 & 73.00 \\
\hline
\end{tabular}


show all $(n=64)$ experiment results for $B=0.05,0.1,0.15$ and 2.0. Annotations $\mathbf{0}$ to 6 indicate the reflector profiles shown in a later section.

\subsection{Generalized geometric factor $G$ and synthesis response $S$}

Factors for describing the structure of the reflector depended on the sensitivities to the overall performance properties and the appropriate mathematical definitions. One can now investigate the effects of these factors to energy and uniformity by the principle component analysis (PCA) $[14,15]$. Table 5 gives the correlation coefficients between the three geometric factors $(B, C, D)$ and the two optical metrics $(P, U)$. Results indicated that the angle factor $C$ has a positive correlation with the total power $P$, while the two factors $B$ and $D$ contribute to the uniformity $U$. In all the experiments, negative correlation between $P$ and $U$ was around -0.82106 , and this negative correlation meets the physical senses of the reflectors, without any secondary lens optics. Finally, during the design of reflectors (or any other types of optical system), one could ask if it is possible to just use one representative factor and one synthesis response to represent the general correlations of the entire optical system.

$\mathrm{T}$ a b l e 5. Correlation coefficients between the shape factors $(B, C, D)$ to the performance metric $(P, U)$.

\begin{tabular}{|c|c|c|c|c|c|}
\hline & B & $C$ & $D$ & $P$ & $U$ \\
\hline $\bar{B}$ & 1 & 0 & 0 & -0.66114 & 0.48798 \\
\hline C & & 1 & 0 & 0.48009 & -0.48811 \\
\hline$D$ & & & 1 & -0.46192 & 0.54158 \\
\hline$P$ & & & & 1 & -0.82106 \\
\hline$U$ & & C & & & 1 \\
\hline
\end{tabular}

In this paper, the goal is to find the combination of the three geometric factors $(B, C, D)$ into a generalized geometric factor $G$, and the transformation of the two performance metrics $(P, U)$ into a synthesis performance $S$, such that one can build a correlation between $G$ and $S$ for extracting the essential properties of the reflector. To achieve this, this report performs a canonical analysis $[\underline{13}-15,19]$ for all 64 sets of experimental results. After that, two generalized factors (called the roots in canonical analysis) for $G$ and two roots for $S$ are obtained. However, the $\chi^{2}$ test results show that only the factor with a canonical correlation $R_{\mathrm{P}}=0.95299$ meets the $H_{0}$ hypothesis. This new factor $G$ can be applied as the generalized geometric factor of the reflector (canonical correlation for the impractical one is 0.47143 ), and synthesis response $S$ is

$$
\begin{aligned}
& G=0.664 B^{*}-0.527 C^{*}+0.531 D^{*} \\
& S=-0.721 P^{*}+0.319 U^{*}
\end{aligned}
$$

where $\left(B^{*}, C^{*}, D^{*}\right)$ and $\left(P^{*}, U^{*}\right)$ are the normalizations of canonical analysis for the $(B, C, D)$ and $(P, U)$ through 


$$
\begin{aligned}
B^{*} & \equiv \frac{B-\bar{B}}{\sigma_{B}} \\
C^{*} & \equiv \frac{C-\bar{C}}{\sigma_{C}} \\
D^{*} & \equiv \frac{D-\bar{D}}{\sigma_{D}} \\
U^{*} & \equiv \frac{U-\bar{U}}{\sigma_{U}} \\
P^{*} & \equiv \frac{P-\bar{P}}{\sigma_{P}}
\end{aligned}
$$

where $\bar{B}, \bar{C}, \bar{D}, \bar{U}, \bar{P}$ and $\sigma_{B}, \sigma_{C}, \sigma_{D}, \sigma_{U}, \sigma_{P}$ are the means and deviations for $B, C$, $D, U$ and $P$, respectively.

\subsection{Structure equation}

Equations (6) demonstrate that the uniformity $U$ and power collections $P$ are in contradiction to each other for the synthesis response $S$. It also indicates that for this configuration (Fig. 2), the effect of the angle factor $C$ is on the negative phase to other two geometric factors $B$ and $D$, during the design of the reflector. Now, Fig. 5 provides the scattering plot between the factors $G$ and the response $S$ for the complete 64 experimental configurations. Interestingly, the canonical correlation coefficient $R_{\mathrm{P}}$ between $S$ and $G$

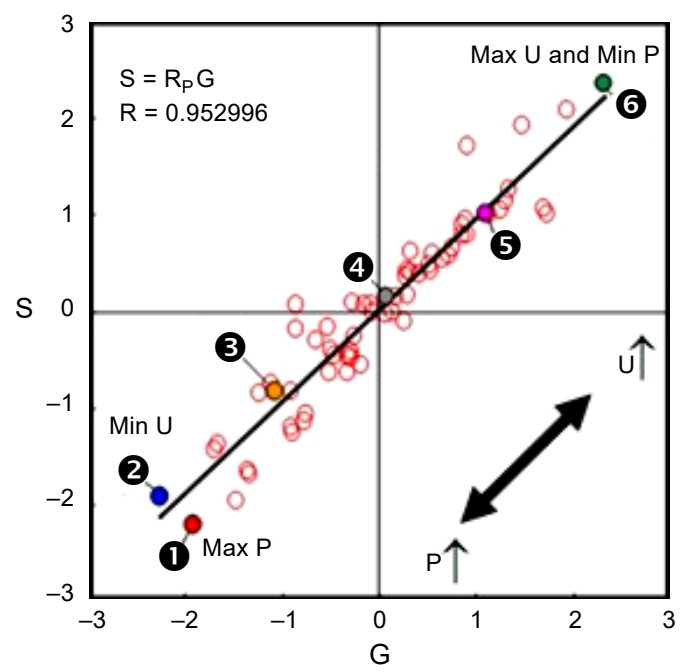

Fig. 5. Generalized geometric factor $G$ and synthesis response $S$ by canonical analysis. 


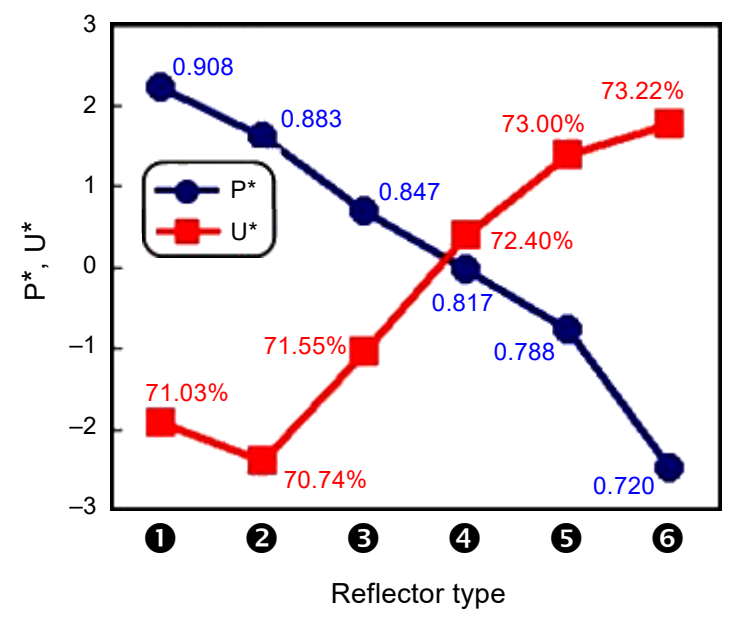

Fig. 6. Uniformity $U$ and power $P$ as well as their normalized quantities $U^{*}$ and $P^{*}$ for six specific reflector profiles indicated in Fig. 5.

reaches 0.952996 , which indicates a good linear regression relationship for the optical characteristics of the reflector. The importance of Eqs. (6), Fig. 5 and the $R_{\mathrm{P}}$ coefficient is that together they bring up a beautiful linear structure equation [15] of the reflector:

$$
S=R_{\mathrm{P}} G
$$

Equation (8) provides a simple and effective description for designing reflector performance by the geometry parameters $(B, C, D)$. In addition, this structure equation indicates the dependent properties between the power $P$ and the uniformity $U$, such that the designed merit function $M$ for optimization is actually constrained by it. This can become the guidelines for reducing the expenditure on non-imaging optimizations since any feasible solutions must follow this reflector structure equation (in the average). The concept of structural equation can also be extended to other optical components, such as the secondary lens or Fresnel-like prism [20]. The right-upper part of Fig. 5 indicates the reflector shape design for the better uniformity, while the left-lower part is for the maximum power collection design. As speculated, that particular design is happening to be near the $G \approx 0$ and $S \approx 0$ configuration, which represents the balancing on both the uniformity and the energy collection capabilities of the reflector, with particular angular apodization shown in the latter. In this figure, six different reflector profiles are chosen for further investigations and comparisons in a later section. At the same time, Fig. 6 provides the transient of the power and the uniformity between these reflector shapes under the normalized metric $P^{*}$ and $U^{*}$. For conveniences, this figure is annotated by the original metric $P$ and $U$. Pay attention that shape $\mathbf{0}$ is serving for maximum power $(\operatorname{Max} P)$, shape 2 is denoted as the minimum uniformity (Min $U$ ), and shape $\boldsymbol{\sigma}$ demonstrates the maximum uniformity/minimum power collection per- 
$\mathrm{T}$ a b 1 e 6. Geometric parameters and optical performance metrics for six reflector shapes; $\theta=55^{\circ}$ $(C=1.42815)$, and $\theta=40^{\circ}(C=0.83910)$.

\begin{tabular}{lllllllll}
\hline Shape & $B$ & $C$ & $D$ & $P$ & $U[\%]$ & \multicolumn{1}{l}{$P^{*}$} & \multicolumn{1}{l}{$U^{*}$} & \multicolumn{1}{l}{$S$} \\
\hline ( & 0.05 & 1.42815 & 2.85714 & 0.908 & 71.03 & 2.216 & -1.914 & -2.208 \\
2 & 0.05 & 1.42815 & 2.5 & 0.883 & 70.74 & 1.606 & -2.400 & -1.924 \\
3 & 0.15 & 1.42815 & 2.5 & 0.847 & 71.55 & 0.694 & -1.042 & -0.833 \\
4 & 0.10 & 0.83910 & 2.85714 & 0.817 & 72.40 & -0.040 & 0.383 & 0.151 \\
5 & 0.15 & 0.83910 & 3.33333 & 0.788 & 73.00 & -0.779 & 1.389 & 1.005 \\
6 & 0.20 & 0.83910 & 4 & 0.720 & 73.22 & -2.475 & 1.758 & 2.346 \\
\hline
\end{tabular}

formance (Max $U$ and Min $P$ ). The chosen for the profiles $\mathbf{3}, \mathbf{4}$ and $\mathbf{6}$ are for the parameter $G$ close to $-1,0$ and 1 .

The implementation of the structure equation is easy and effective. For an LED fabricator, a testing environment (or a benchmark device) that is similar to Fig. 2 with position of sensors shown in Fig. 4 can be established easily. For a series of LED product, factor $B$ and the properties of LED die are usually predetermined. The geometric factors $(B, C, D)$ and the performance metric $(P, U)$, along with their normalized representation $\left(P^{*}, U^{*}\right)$ and the synthesis performance $S$, are given in Table 6 (recall that $\left.S=-0.721 P^{*}+0.319 U^{*}\right)$.

Once the structure equation is suggested, the following design protocol provides a novel methodology to evaluate and complete the optimization of reflector designs, without the requirements on cumbersome ray tracing:

1. Assign any one of the factors (for example, $B$ ) by the LED fabricator as control parameters;

2. Take the rest two geometric factors $(C, D)$ to form the initial design space;

3. Obtain the synthesis factor $G$ for each $(B, C, D)$ set by Eq. (6);

4. Using the structure equation (8) to obtain the predicted response $S$;

5. For targeting uniformity $U$, a corresponding power collection $P$ can be predicted by Eq. (6);

6. User defined merit function $M=M\left(P, U ; w_{i}\right)$ that consists of the performance metric $(P, U)$ with appropriate weighting $w_{i}$ for a particular application can be constructed for proceeding the optimization.

7. Fabricators can obtain the response surface of the merit function $M(C, D)$ by taking $(C, D)$ as variable space.

8. Fabricators can also investigate the variations of the response surface $M$ by considering the factor $B$ and the weightings $w_{i}$ as control parameters for exploring the optimum design (i.e., $M=M\left(C, D ; B, w_{i}\right)$ ).

Fewer experiments might lose the accuracy but still can produce a reliable structure equation [13-15]. Moreover, there will be no need for the optical designer to run hundreds times of non-imaging performance evaluations under millions rays tracing, once the structure equation $S=R_{\mathrm{P}} G$ is obtained - this is of great cost effective for fabricators. 


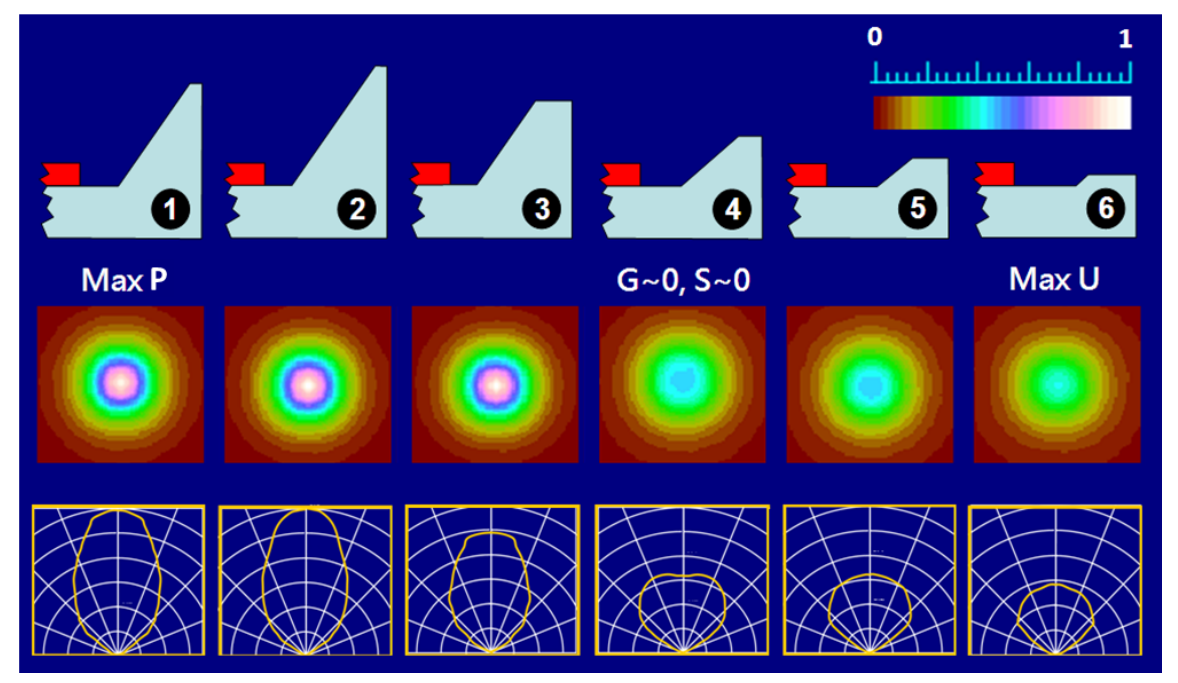

Fig. 7. Comparison of six reflector shapes, illuminating patterns and spatial apodization for different reflector types with specific geometric parameters.

The present canonical analysis procedures, the definitions of the performance and the optimization methodology are not limited to reflector design, but can also be applied to all kinds of optical components and performance metrics.

\subsection{Profiles and performance of reflectors}

Based on the structure equation $S=R_{\mathrm{P}} G$ and the previous discussions on the performance metrics, in this section the geometric profiles for these six reflectors are shown together in Fig. 7 for the comparison of the profiles. To understand the performance of these reflector shapes, Fig. 7 also demonstrated the illumination distributions and angular apodizations with normalized intensity scale bars, and this figure addresses the annotations with $\operatorname{Max} P$, Max $U$ and $G \approx 0, S \approx 0$ for three specific profiles. Through the inspection between spatial apodization/reflector profiles and illuminating patterns, three intuitive summaries can be made in this section. Firstly, it is interesting to see that the profiles for extreme optical performance conditions (shape $\mathbf{0}, \boldsymbol{2}$ and $\boldsymbol{6}$ ) are almost in the different geometric design space - smaller $B, D$ and larger $C v s$. larger $B, D$ with smaller $C$. Secondly, there is a larger energy (power under steady state) distribution region for shape $\boldsymbol{\sigma}$, while there are more concentrated illuminating patterns for shapes $\boldsymbol{0}$ and $\boldsymbol{2}$. The peak illumination and intensity for the shape $\boldsymbol{\sigma}$ is around only half of the shape $\mathbf{0}$ and 2 . Finally, pay attention to the angular distribution of the shape type number 4 . A lower intensity in the 0 degree than the \pm 25 degree is observed, which forms a slightly $\mathrm{V}$ type angular apodization to balance the uniformity and the collected power. More aggressive $\mathrm{V}$ type apodization is possible for other geometric combinations, which is the direct result of the interaction between the high of the LED die $h_{\mathrm{LED}}$ and the reflector factors. 


\section{Conclusions}

This report discusses the designing of the geometric factors of the reflector to the two demonstrated performance metrics - uniformity $U$ and collected power $P$. Three geometric factors $B, C, D$ are proposed, and a methodology by canonical factor analysis for designing the optimal shape is discussed. Moreover, a generalized geometric factor $G$ and synthesis response $S$ are proposed to describe the reflector system and make it possible to reduce the modelling parameters. Based on this, a particular linear structural equation $S=R_{\mathrm{P}} G$ is suggested for the reflector design, which can greatly reduce the analysis cost for exploring the behavior of the LED/reflector module.

Acknowledgements - Financial support for this research from the Ministry of Science and Technology (MOST-Taiwan) under grant Nos. MOST 104-2221-E-164-008, MOST 104-2633-E-164-001, MOST 105 -2221-E-164-004, MOST106-2221-E-164, MOST106-EPA-F-017-001 and MOST 107-2221-E-164-002 are most appreciated. Author also would like to appreciate the cooperation with Z.W. Huang, R.Y. Lan, Y.J. Hsu and C.Y. Ou for IDW2016, as well as Prof. C.H. Lin and Prof. S.L.Young for IDW2012 technical abstract.

\section{References}

[1] Wang S., Wang K., Chen F., Liu S., Design of primary optics for LED chip array in road lighting application, Optics Express 19(S4), 2011, pp. A716-A724, DOI: 10.1364/OE.19.00A716.

[2] http://www.lighting.philips.co.uk (accessed 2019).

[3] GoRdon J.M., RABL A., Reflectors for uniform far-field irradiance: fundamental limits and example of an axisymmetric solution, Applied Optics 37(1), 1998, pp. 44-47, DOI: 10.1364/AO.37.000044.

[4] Bäuerle A., Schnitzler C., Wester R., Kirsten M., Schlüter H., Tewes M., Laschefski H., Loosen P., Optical system design for a reflector-based LED food lighting module, Proceedings of SPIE 8123, 2011, article 812306, DOI: 10.1117/12.893688.

[5] Jafrancesco D., Mercatelli L., Fontani D., SAnsoni P., Optical CaD utilization for the design and testing of a LED streetlamp, Materials 10(9), 2017, article 985, DOI: 10.3390/ma10090985.

[6] https://www.lumileds.com/uploads/227/AB20-5-PDF (accessed 2019).

[7] CHEN E., Yu F., Design of LED-based reflector-array module for specific illuminance distribution, Optics Communications 289, 2013, pp. 19-27, DOI: 10.1016/j.optcom.2012.09.082.

[8] Tran N.T., SHI F.G., LED package design for high optical efficiency and low viewing angle, [In] 2007 International Microsystems, Packaging, Assembly and Circuits Technology, 2007, pp. 10-13, DOI: $10.1109 /$ IMPACT.2007.4433557.

[9] Ou C.R., Ho R., Kung C.Y., Energy distribution issues for the designing of the LED reflector, [In] Proceedings of 1st International Conference on Display LED (ICDL 2007), Seoul, Korea, pp. 103-105.

[10] Ma M., Mont F.W., Yan X., Снo J., Schubert E.F., Kim G.B., Sone C., Effects of the refractive index of the encapsulant on the light-extraction efficiency of light-emitting diodes, Optics Express 19(S5), 2011, pp. A1135-A1140, DOI: 10.1364/OE.19.0A1135.

[11] Huang Y., Huang Z., Zhong Z., Yang X., Hong Q., Wang H., Huang S., Gao N., Chen X., Cai D., KANG J., Highly transparent light emitting diodes on graphene encapsulated Cu nanowires network, Scientific Reports 8, 2018, article 13721, DOI: 10.1038/s41598-018-31903-7.

[12] Montgomery D., Design and Analysis of Experiments, 5th Ed., Wiley, 2001.

[13] http://www.statsoft.com (accessed 2019).

[14] Jolliffe T., Principal Component Analysis, Springer-Verlag, 1986, p. 487.

[15] Hair J.F., Jr., Anderson R.E., Tatham R.L., Black W.C., Multivariate Data Analysis, 5th Ed., Prentice Hall, 1998, Chap. 8. 
[16] Ou C.-J., Lin C.-H., Young S.-L., Analysis of light emitting diode reflector by factor analysis, [In] Proceedings of 19th International Display Workshops in conjunction with Asia Display 2012 (AD/IDW 2012), Kyoto, Japan, pp. 1538-1539.

[17] Light-Tool 8.3 Illumination Module User's Guide, Optical Research Associates, 2015, p. 141.

[18] http://www.ansys.com (accessed 2019).

[19] Ou C.-J., Huang Z.-W., Hsu Y.-J., Lan R.-Y., Ou C.-Y., Structural equation approach for the designing of LED cup reflector and the future prospect, [In] Proceedings of 23th International Display Workshops in conjunction with Asia Display 2016 (AD/IDW 2016), Fukuoka, Japan, pp. 398-399.

[20] Ou C.-J., Fresnel-like prism structure generating algorithm on a curved surface with iterative and non-iterative forms, Journal of the Optical Society of America A 29(7), 2012, pp. 1427-1435, DOI: 10.1364/JOSAA.29.001427.

Received January 6, 2019 in revised form March 31, 2019 